This article is a POSTPRINT of a paper published in Radiotherapy and Oncology

(that is, it is the authors' version before final acceptance for publication).

Please obtain and cite the final version direct from the journal.

Suggested citation:

Lehman M, Gorayski P, Watson S, Edeling D, Jackson J, Whitty JA. Patient preferences regarding prophylactic cranial irradiation: A discrete choice experiment. [In Press. Accepted Radiotherapy and Oncology 12 Sept 2016] 


\section{Patient Preferences Regarding Prophylactic Cranial Irradiation: A Discrete Choice Experiment}

Lehman M MBBS FRANZCR GDPH ${ }^{a, b}$, Gorayski P BSc BMBS FRACGP FRANZCR ${ }^{b, c}$, Watson S RN ${ }^{a}$, Edeling D RN ${ }^{a}$, Jackson J MBBS FRANZCR ${ }^{d}$, Whitty J.A. PhD ${ }^{\text {e }}$

(a) Department of Radiation Oncology, Princess Alexandra Hospital, Brisbane, Queensland

(b) School of Medicine, University of Queensland

(c) Department of Radiation Oncology, Radiation Oncology Centres, Mater Private Hospital Springfield, Springfield Central, Queensland

(d) Department of Radiation Oncology, Radiation Oncology Centres, Gold Coast University Hospital, Gold Coast, Queensland

(e) Norwich Medical School, University of East Anglia, Norwich, UK

Corresponding Author:

Associate Professor Margot Lehman

Department of Radiation Oncology

Princess Alexandra Hospital

Ipswich Road

Woolloongabba Q 4102

Email: Margot.Lehman@health.qld.gov.au

Conflicts of Interest: None

Acknowledgement: The authors would like to thank Dr Redzo Mujcic for providing helpful comments on an earlier version of this paper. 


\section{Abstract}

\section{Introduction:}

In patients with Non-small cell lung cancer (NSCLC) treated with chemoradiotherapy (CRT), prophylactic cranial irradiation (PCI) is not standard practice. This study determined patient preferences for $\mathrm{PCl}$ with respect to survival benefit, reduction in brain metastases (BM) and acceptable toxicity.

\section{Methods:}

A Discrete Choice Experiment was completed pre- and post- treatment. Patients made 15 hypothetical choices between two alternative PCl treatments described by four attributes: amount of life gained, chance of BM, ability to care for oneself, and loss of memory. Participants also chose between PCl and no PCl.

\section{Results:}

54 and 46 surveys were completed pre- and post-treatment. Pre-treatment the most important attributes were: a survival benefit $>6$ months, of $3-6$ months, avoiding severe problems with memory and self-care, avoiding quite a bit of difficulty with memory and maximally reducing BM recurrence. Posttreatment, BM reduction became more important. $90 \%$ of patients would accept PCl for a survival benefit $>6$ months, with a maximal reduction in BM even if severe memory/self-care problems occurred. With a $10 \%$ reduction in BM and mild problems with memory and self-care $70 \%$ of patients pre- $(90 \%$ posttreatment) would accept PCI for a survival benefit of 1-3 months, and $52 \%$ pre- (78\% post-treatment) for no survival benefit.

\section{Conclusion:}

Improvement in survival is the most important attribute of $\mathrm{PCl}$ with patients willing to accept significant toxicity for maximum survival and less toxicity for less survival benefit. BM reduction became more important after treatment. The majority of patients would accept PCl for no survival benefit and a reduction in $\mathrm{BM}$. 


\section{INTRODUCTION}

Brain metastases (BM) develop in 22-55\% of patients with locally advanced Non-small cell lung cancer (LA-NSCLC) treated with curative intent. ${ }^{[1,-4]}$ Technological advances in tumour staging, radiation therapy (RT) planning and delivery, and the concurrent administration of chemotherapy with RT, has translated into improved local control and survival rates. ${ }^{[5,6]}$ However, improvements in survival provide a longer time period for micro-metastatic disease to manifest and it is hypothesised that the incidence of BM will increase.

In selected patients with limited- and extensive- stage small cell lung cancer (SCLC), the use of $\mathrm{PCl}$ is considered standard of care as $\mathrm{PCl}$ results in a decreased incidence of BM and an improvement in disease-free survival (DFS) and overall survival (OS). ${ }^{[7-9]}$ In LA- NSCLC, PCI is not part of standard practice due to a lack of proven survival benefit and concerns regarding neurotoxicity. The Radiation Therapy Oncology Group (RTOG) 0214 study randomised patients with Stage III NSCLC treated with CRT to PCI or observation. PCl reduced the incidence of BM at 1 year $(18 \%$ v $7.7 \%, p=0.004)$ but was not associated with an improvement in DFS or OS. However, the study was underpowered for a survival endpoint. ${ }^{[10]}$ The use of PCl was associated with some adverse effects, namely a decline in delayed recall on the Hopkins Verbal Learning Test (HVLT). However, there were no differences observed between the study arms on Mini Mental Status Examination, Activities of Daily Living scale or quality of life (QOL) assessments. ${ }^{[1]]}$

The present study aimed to determine the preferences of patients with LA-NSCLC undergoing CRT with respect to PCI. Specifically, this study aimed to (i) estimate the relative importance of different characteristics potentially associated with $\mathrm{PCl}$ (survival gains, reduced chance of BM, reduction in ability to care for oneself and memory loss) for patients; (ii) estimate the impact of changes of these characteristics on the likelihood that patients would accept PCl, and (iii) explore whether undertaking CRT for lung cancer has an impact on patients preferences for $\mathrm{PCl}$.

\section{METHODS}

A Discrete Choice Experiment (DCE) was used in a prospective cohort of patients with LA-NSCLC undergoing CRT at a single tertiary institution in Queensland, Australia, between June 2012 and December 2014. The DCE is a popular method for quantifying and evaluating patient preferences in healthcare. ${ }^{[12,13]}$ In a DCE, a medical intervention is described by its attributes (e.g. improvement in survival, or adverse effects). A series of hypothetical choice sets is constructed in which the levels of these attributes change in a systematic way (according to an underlying experimental design). In each choice set, the participant is asked to perform a pairwise comparison of these hypothetical alternatives and choose their preferred alternative. Thus, participants exercise their preferences by making repeated trade-offs and choices across these hypothetical scenarios.

\section{Attributes and Attribute Levels}


The choice of the most relevant attributes and levels of PCI for LA-NSCLC was based on literature findings. ${ }^{[10,11]}$ The attributes chosen were: amount of life gained, chance of developing BM, ability to care for oneself and loss of memory. Each attribute was described using four levels that were felt to represent potential outcomes from PCl. Each level was presented in a way that was felt to be meaningful to patients. For example, to assess the importance of developing BMs, patients were asked to choose between different "chances of cancer coming back in your brain" with the chances given as $20 \%$; 4 in 20 patients, (to approximate the 18\% incidence of BM in the observation arm of RTOG 0214), 10\%; 2 in 20 patients (to approximate the 7.7\% incidence of BM in the treatment arm of RTOG 0214) with an intermediate level (15\%; 3 in 20 patients) and a best case scenario level (5\%; 1 in 20 patients). For amount of life gained, levels ranged from no extra life (reflecting ROTG 0214 results) to a hypothetical best case scenario of $>6$ months of life gained, with intermediate levels of 1-3 and 3-6 months of extra life. Similarly, to assess the importance of impact on memory and self-care, levels ranged from no problem or difficulty to severe problems or difficulties. The attributes and the associated levels are presented in Table 1.

\section{DCE choice set design and survey}

NGENE software (ChoiceMetrics, 2012) was used to combine the attributes and levels into 16 choice sets, using a D-optimal design approach. ${ }^{[14]}$ This ensured a statistically efficient approach was used to capture the choice data. In each choice set the presented attribute levels were varied in order to capture the different trade-offs which individuals usually face with respect to their radiation treatment options. Participants were initially asked to choose between two alternative PCl treatments in each choice set. They were then asked to choose between their preferred PCl alternative and an opt- out alternative of no PCl. The opt- out alternative was described as providing no extra life, a $20 \%$ risk of $\mathrm{BM}$, and no change in ability to take care of oneself or memory. Figure 1 presents an example choice situation.

To assess whether the experience of undergoing conventional therapy impacted on participant choices, the DCE was undertaken two time points,: (1) just prior to commencement of CRT or during the first week of therapy; and (2) during the last week of therapy. In addition, the impact of patient

sociodemographic factors and self-reported health status (obtained from the EQ-5D-5L instrument) on patient preferences was assessed. ${ }^{[15]}$ The survey was pilot tested in an interview-based setting prior to data collection, to ensure the survey was understood by patients and had face validity.

\section{Sample and administration}

Ethical approval was obtained from the hospital Human Research Ethics Committee. Patients were eligible if they were diagnosed with Stage III NSCLC and were to receive definitive CRT. Patients were identified at the weekly Lung Cancer Multi-disciplinary Meeting. Once consent had been obtained, the DCE was administered by trained nurses. The nurse presented each alternative to the participant in a face-to-face interview setting using a booklet, and recorded their choice. Participants were informed that the scenarios were hypothetical.

\section{Data analysis}


The choice data were analysed using mixed logit regression (MXL) models to indicate the relative importance of improvements in each attribute for treatment preference. ${ }^{[16]}$ In a mixed logit model, the participant's choices are related to the corresponding treatment attribute levels and a set of socioeconomic individual characteristics. Such an approach allows an estimate of the relative importance of each treatment attribute, in addition to the implied trade-offs between selected attributes, i.e. how much of one attribute a participant is willing to forgo in order to gain a fixed amount of another. It also allows an estimate of likely uptake for an intervention.

The MXL model was specified as a linear function of the attribute levels. The constant term associated with treatment uptake was treated as random (following a normal distribution, using 1000 Halton draws for estimation) and dependent on other sociodemographic variables including the participant's age, education level, employment status, marital status, and self-reported health status. All attribute levels were effects coded, except for BM which was coded continuously. The opt out alternative of no PCl was specified with a fixed utility equal to that associated with no extra survival and a $20 \%$ chance of $\mathrm{BM}$, as these were the levels used to describe the setting of "no PCl" in the DCE survey.

The size of the coefficients in the models indicates the importance of improvements in different PCl treatment attributes. To estimate the relative importance of improvements in different attributes for participants and enable their comparison between the pre- and post-treatment models, the model coefficients were weighted for each model such that a $1 \%$ reduction in the chance of BM was given 1 point, and the other improvements were given a greater number of points in proportion to their relative importance for participants. The probability of uptake of PCl by participants, relative to not taking up PCl, was also estimated from the model based on the coefficients. This was estimated for selected scenarios representing a range of outcomes across the spectrum of attributes considered to be of particular relevance to clinical practice and the current evidence base for $\mathrm{PCl}$ treatment.

\section{RESULTS}

There were 54 and 46 surveys completed pre- and post-treatment, respectively. The sample population appears representative of the LA-NSCLC patient population. Participants had a mean age of 63.6 years (SD 10.1, range 39-82), and $74 \%$ were male. High-school was the highest educational level for $48 \%$ of patients. The mean self-reported visual analogue score on the EQ-5D-5L was 74.3 out of 100 (SD 17.6, range 30 to 100). Participant characteristics are reported in Table 2.

There were 807 choice observations available for analysis pre-treatment (54 participants x 15 choice sets less 3 missing responses) and 687 post-treatment (46 participants $x 15$ choice sets less 3 missing responses). One of the 16 choice sets was omitted in error from the post-treatment survey; therefore, only the 15 choice sets that were completed in both the pre- and post- surveys were included in the models. Estimation results from mixed logit models are reported in Table 3. Both models represented a reasonable fit for the choice data (pseudo-r squared 0.35-0.36), ${ }^{[17]}$ and the estimated coefficients on the treatment attributes had the expected signs. For example, the coefficients for the 'amount of extra life gained' attribute levels were larger than the referent level of no extra life and statistically significant, broadly indicating that if the survival rate in one treatment alternative increases, then preference for that treatment alternative increases. The only counterintuitive result was for the attribute "taking care of oneself" for which the post-treatment model indicated 
a significant preference for "slight problems" over "no problems". Overall, participants preferred a treatment alternative if it was associated with a longer survival, better ability to take care of oneself, lower loss of memory, and lower chance of occurrence of BM.

The importance weights of the $\mathrm{PCl}$ attributes both before and after treatment, implied by the models are presented in Table 4 and Figure 2. Before treatment, an increase in survival of more than 6 months and of 3 to 6 months were the most important benefits (relative importance weights 61.2 and 42.2 respectively), followed by avoiding severe problems with memory (39.4), avoiding severe problems with self-care (30.4), avoiding quite a bit of difficulty with memory (26.2), and a $15 \%$ reduction in risk of BM (15.0). After treatment, there were some differences in the rank order of importance with avoiding severe problems with self-care becoming more important than a 3-6 month gain in extra life, and avoiding severe problems with memory becoming less important. However, a reduction in the risk of BM became more important post-treatment, relative to improvements in the other attributes. That is, following treatment, participants appear to care more about the risk of cancer returning to their brain relative to the benefit of gains in other attributes, and are more willing to accept a reduced ability to care for oneself and increased memory loss, in order to achieve a reduced risk of BM. However, any comparison between the pre- and post-treatment survey is indicative only, due to the limited sample size.

Participants chose to take up PCl in approximately two thirds of the raw choice sets (65.2\% or 526/807 pre; $66.7 \%$ or $458 / 687$ post; $65.9 \%$ or $984 / 1494$ overall). At the individual level, participants opted out of treatment by choosing "no PCl" for a mean of 5.2 (SD 5.2; median 3.0 IQR 0.75 to 10.0 ; range 0 to 15) choice sets pre-treatment and 5.0 (SD 5.4; median 3.0, IQR 0.0 to 9.0; range 0 to 15) choice sets post-treatment.

From the pre-treatment MXL model (Table 3), participants with a lower education level (highest level of education high-school) were on average more likely to consider $\mathrm{PCl}$, all else equal, than those with a higher level of education $(p<0.01)$. On the other hand, participants with better health levels (EQ-5D VAS score 275 ) were less likely to consider PCl compared to participants with lower levels of self-reported health; although this only reached significance at the $10 \%$ level. None of the other sociodemographic characteristics (age, gender, employment status, marital status, carer status) were associated with the choice to take up $\mathrm{PCl}$, either pre- or post-treatment; although this lack of association should be interpreted with caution, given the sample size. The estimated standard deviation corresponding to the constant term is large and statistically significant (at the $1 \%$ level) both pre- and post-treatment, indicating notable variation in tastes across individuals in their choice to accept $\mathrm{PCl}$ which is not explained by the included individual characteristics.

The probability of uptake of $\mathrm{PCl}$ under a range of scenarios (in which the levels of the characteristics varied), relative to no PCl, both before and after $\mathrm{CRT}$ is presented in Tables 5 and 6 . In the best case scenario of PCl providing >6 months extra life, a 15\% reduction in chance of BM (from $20 \%$ to $5 \%$ ) with no associated problems with self-care or memory, almost all participants (96\%) would accept PCI. If PCl increased survival by $>6 \mathrm{~m}$ and reduced $\mathrm{BM}$ risk by $15 \%$ (from $20 \%$ to $5 \%$ ) but resulted in severe problems with self-care or memory loss, $63 \%$ of participants would accept PCl pre-treatment and $76 \%$ posttreatment.

In a scenario of $\mathrm{PCl}$ providing a small survival benefit (1-3m), a small reduction in BM (5\% from $20 \%$ to $15 \%)$ and resulting in moderate problems with selfcare and quite a bit of difficulty with memory, $45 \%$ of patients pre-treatment and $71 \%$ of patients post treatment would accept $\mathrm{PCl}$. In a scenario of no 
survival benefit or reduction of $\mathrm{BM}$ risk with $\mathrm{PCl}$, as long as patients were left with no adverse effects (defined as no problems with self-care or memory); over half of patient would accept $\mathrm{PCl}$ ( $57 \%$ before and $69 \%$ post conventional treatment).

The factors that would prompt the vast majority of patients to take up PCl are presented in the last 4 columns of Table 5 . Over $90 \%$ of participants would accept PCl if it gave at least a $10 \%$ reduction in absolute BM risk and 3 to 6 months survival benefit, as long as it was associated with no problems with selfcare or memory. Over $80 \%$ would accept PCl if it gave at least a $10 \%$ reduction in the incidence of BM and 1-3m survival benefit, as long as there was no detrimental effect on self-care or memory.

RTOG 0214 demonstrated that, at 1 year, $\mathrm{PCl}$ reduced the incidence of BM from $18 \%$ to $7.7 \%$ with no associated survival benefit and with a decline in memory (as measured by the HVLT). We undertook hypothetical modelling using these findings and altering the survival benefit to investigate the uptake of PCI (Table 6). Assuming PCl reduced the incidence of BMs by $10 \%$ (from $20 \%$ to $10 \%$ ) and left patients with mild problems with self-care and memory over $90 \%$ of participants would accept PCl if it provided over 6 months survival benefit , $84 \%$ if survival benefit was 3 to 6 months (rising to $93 \%$ posttreatment), $71 \%$ for 1 to 3 months survival benefit (rising to $90 \%$ post-treatment) and $52 \%$ (rising to $78 \%$ post-treatment) would accept PCl even if there was no known survival benefit.

\section{DISCUSSION:}

Given that a significant proportion of patients with LA-NSCLC undergoing CRT develop BMs which negatively impacts neurocognitive function, QOL, and prognosis, there are compelling reasons why attempts to prevent BM have been investigated. ${ }^{[18]}$ Six randomised controlled trials have evaluated the role of $\mathrm{PCl}$ in LA-NSCLC, with five studies demonstrating a statistically significant reduction in the incidence of BM with PCl, but no survival advantage. ${ }^{[10,19-23]}$ The most recent of these studies was underpowered for a survival endpoint. ${ }^{[10]}$ It is not clear whether patient refusal was the reason why target accrual was not met.

The aims of this study were to estimate what factors were important from the patients' perspective with regard to the use of PCl. Specifically, we wished to address the relative importance of the different characteristics potentially associated with $\mathrm{PCl}$ (reduced chance of BM, impact on survival, reduction in ability to care for herself, memory impairment); the impact that changes in any of these characteristics would have on the patients' preferences for PCl; and the impact of completing conventional treatment for LA-NSCLC on patients' preferences. In this way, we wished to provide information which could inform decisions as to whether evaluating newer strategies, which aim to reduce the neurocognitive impact of whole brain radiotherapy, would be acceptable to patients.

We found that participants would choose to undergo PCl in approximately two thirds of the hypothetical scenarios. Participants with a higher educational level and, to a lesser extent, better health status were more likely not to choose PCI. We hypothesise that patients with a higher level of educational 
achievement place more value on their neurocognitive abilities and therefore may be more concerned about the potential impact of $\mathrm{PCl}$ on their ability to function. An alternative explanation that participants with a lower educational level did not understand the DCE concept cannot be discounted. However, the survey was pilot tested in an interview-based setting prior to data collection, to ensure the survey was understood by patients. In addition, the trained nurses who administered the DCE reported that a lack of understanding of the task was not an issue. Similarly we hypothesise that patients with a better health status pre-treatment and who are desirous of returning to that level post-treatment, are more likely to be concerned about side effects that could have long term impact on their health status and ability to function.

We found that improvement in survival is the most important potential characteristic of $\mathrm{PCl}$ for patients both pre and post-treatment and patients indicated they were willing to accept significant toxicity for maximum survival benefit. If PCl resulted in a 6 month increase in survival and a $15 \%$ reduction in BM but resulted in severe problems with memory or ability to self-care, $63 \%$ of patients pre-treatment and $76 \%$ post-treatment would accept PCl. The degree of toxicity deemed acceptable became less as survival benefit decreased, but a moderate amount of toxicity was still acceptable to a significant proportion of patients. For a survival benefit of 1-3 months, associated with a $5 \%$ reduction in BM, $45 \%$ of patients pre-treatment and $71 \%$ post-treatment would accept $\mathrm{PCl}$, even if it was associate with moderate problems with self-care and quite a bit of difficulty with memory. It could be hypothesised that the patients family or caregivers would have an impact on the trade-offs patients made between survival gain and potential impact on memory and self-care. However, while we found that the patients marital or carer status did not influence their choice to accept PCl over no PCl, a result that should be interpreted with caution due to the small sample size, we did not examine the influence of family or carers on individual trade-offs. This would be an interesting subject for further evaluation.

While a potential survival gain was the most important characteristic of $\mathrm{PCl}$, we found that patient's preferences changed upon completion of treatment with a reduction in BM becoming more important relative to gains in other attributes. That is, following treatment, participants appear to care more about the risk of cancer returning to their brain relative to the benefit of gains in other attributes, and are more willing to accept a reduced ability to care for oneself and increased memory loss, in order to achieve a reduced risk of BM. The explanation for this preference change is uncertain. We hypothesise that as patients approach the completion of their definitive therapy, they become more concerned about preventing disease recurrence in other parts of their body.

When hypothetical scenarios were aligned with the findings of RTOG 0214 (ie an approximate 10\% reduction in BM incidence) over $90 \%$ of patients would accept PCl for a potential survival benefit $>6$ months, $84 \%$ pre- and $93 \%$ post-treatment, for a potential survival benefit of 3-6 months, $71 \%$ pre- and $90 \%$ post-treatment for a potential survival benefit of 1-3 months, and $52 \%$ pre and $78 \%$ post for no survival benefit. These results confirm that while potential improvement in survival is most important, a reduction in BM incidence, particularly after completion of treatment is important for a significant proportion of patients. Furthermore, over $50 \%$ of patients indicated they would accept $\mathrm{PCl}$ if there were no improvement in survival or reduction in $\mathrm{BM}$ as long as there 
was no impact on memory or ability to self-care. This acceptance of treatment by a significant proportion of patients for minimal or no improvement in survival, has previously been documented in literature investigating the treatment preferences of women with breast cancer. ${ }^{\text {[24, 25] }}$

Given that patients diagnosed with BMs have a median survival of 4 months, it seems reasonable that strategies attempting to prevent their occurrence and thereby improve survival continue to be pursued, particularly if such studies enrol patients deemed at high risk of developing BMs and employ techniques which have the potential to reduce the cognitive impact of WBRT. ${ }^{[26]}$ One such technique is hippocampal sparing WBRT (HS-WBRT). HS-WBRT uses advanced RT technology to minimise the radiation dose delivered to the stem cell compartments involved in the neurogenesis of cells responsible for learning and memory functions. HS-WBRT was associated with a significant reduction in mean relative decline in HVLT delayed recall at 4 months in a randomised Phase II trial. ${ }^{[27]}$ A prospective study of patients who examined multi-domains of neurocognitive function before and 4 months after HS-WBRT found neurocognitive function stabilised or improved. ${ }^{[28]}$ Multiple factors including the total RT dose, dose per fraction, concurrent chemotherapy and anticonvulsants, pre-existing cognitive impairment, and the effects of disease-progression have been proposed as impacting on cognitive function ${ }^{[26,29,30]}$ Therefore the impact of HS-WBRT on avoiding neurocognitive decline needs to be assessed in randomised trials. There are currently two co-operative group Phase III trials addressing this question. (NRG-CC 003 and NRG-CCO01). ${ }^{[31,32]}$

Whether HS-WBRT could be safely employed as a strategy for delivering PCl will be dependent not only on the results of the above studies but also of studies investigating the risk and relapse of perihippocampal metastases in NSCLC. Retrospective studies demonstrated the hippocampus to be a rare site of BM occurrence from all tumour histologies. ${ }^{[33,34]}$ A retrospective mapping study of $856 \mathrm{BMs}$ in 100 patients (with the most common histology being NSCLC) found a $2.8 \%$ incidence of NSCLC BMs within the hippocampus, and an $8.3 \%$ incidence in a $5 \mathrm{~mm}$ radius from the hippocampus. ${ }^{[35]}$ Modelling suggested a $0.2 \%$ absolute risk increase due to sub-therapeutic dosing of the hippocampus by HS-WBRT, leading the authors to conclude that HS-WBRT bears a low risk of undertreating patients. ${ }^{[35]}$

It is acknowledged that the results of this study need to be viewed in light of its limitations. Specifically, the small sample size means that any comparison between the pre- and post-treatment survey should be interpreted with caution. In addition, it is acknowledged that cognitive decline affecting memory and self-care can occur as a consequence of both $\mathrm{PCl}$ and $\mathrm{BM}$ and therefore the choice between these attributes is not fully discrete. Furthermore, it is acknowledged that a survival benefit has not been associated with $\mathrm{PCl}$, and thus including potential survival gains as an attribute can be questioned. However, no survival benefit is included as one attribute level. Moreover, the aim of this study was to determine how much hypothetical improvement in survival patients would deem sufficient to accept any potential side effects of therapy. Despite these limitations to the authors' knowledge, this is the first study which has tried to ascertain the preferences of patients who have been diagnosed with LA-NSCLC and who are undergoing CRT with respect to the role of $\mathrm{PCl}$. Furthermore, the use of a DCE methodology, although hypothetical, is widely accepted approach to eliciting patient preferences in healthcare, including radiation oncology ${ }^{[36,37]}$ 
In conclusion, the use of $\mathrm{PCl}$ in unselected patients with LA-NSCLC undergoing curative therapy has resulted in a statistically significant reduction in the incidence of BMs but no proven survival advantage. The results of this study show that, while from the patients' perspective, improvement in survival is the most important potential attribute of $\mathrm{PCl}$, and that the degree of acceptable toxicity is dependent on the amount of life gained, reduction in the incidence of BMs became more important, relative to other attributes after the completion of therapy. Indeed, if $\mathrm{PCl}$ resulted in a $10 \%$ decrease in the incidence of BMs and no survival benefit (as occurred in RTOG 0214), the majority of patients would accept PCI. The results of this study therefore suggest that research protocols assessing the benefit of $\mathrm{PCl}$ in selected patients at high risk of developing $\mathrm{BM}$ and using $\mathrm{PCl}$ techniques with a potential for reducing cognitive impacts, would be acceptable to patients. 


\section{References}

1. Stuschke M, Eberhardt W, Pottgen C, et al. Prophylactic cranial irradiation in locally advanced non-small cell lung cancer after multimodality treatment: long-term follow-up and investigations of late neuropsychologic effects. J Clin Oncol. 1999; 17:2700

2. Carolan H, Sun AY, Bezjak A, et al. Does the incidence and outcome of brain metastases in locally advanced non-small cell lung cancer justify prophylactic cranial irradiation or early detection? Lung Cancer. 2005; 49:109-15

3. Ceresoli G, Reni M, Chiesa G et al. Brain metastases in locally advanced non-small cell lung carcinoma after multimodality treatment: Risk factors analysis. Cancer. 2002; 95:605-612,

4. Marmon HJ, Yeap By, Janne P et al. High risk of brain metastases in surgically staged IIIA non-small cell lung cancer patients treated with surgery, chemotherapy and radiation. J Clin Oncol. 2005; 23: 1530-1537

5. Curran W, Paulus R, Langer C et al. Phase III comparison of sequential v concurrent chemo-radiation for patients with unresected stage III non-small cell lung cancer (NSCLC): report of Radiation Therapy Oncology Group (RTOG) 9410. J Natl Can Inst. 2011; 103: 1452-60.

6. Bradley JD, Paulus R, Komaki et al. Standard-dose versus high-dose conformal radiotherapy with concurrent and consolidation carboplatin plus paclitaxel with or without cetuximab for patients with Stage IIIA or IIIB non-small-cell lung cancer (RTOG 0617): a randomised, two-by-two factorial phase 3 study. Lancet Oncol. 2015. 16: 187-199.

7. Meert A, Paesmans M, Berghams T et al. Prophylactic cranial irradiation in small cell lung cancer: a systematic review of the literature with metaanalysis. BMC Cancer. 2001; 15:1.

8. Auperin A, Arriagada R, Pignon J et al. Prophylactic cranial irradiation for patients with small cell lung cancer in complete remission. Prophylactic Cranial irradiation Overview Collaborative Group. NEJM. 1999; 341: 476-84.

9. Slotman B, Faivre-Finn C, Kramer G et al. Prophylactic cranial irradiation in extensive small-cell lung cancer. N Engl J Med 2007 ; $357: 664-72$.

10. Gore EM, Bae K, Wong SJ et al. Phase III comparison of prophylactic cranial irradiation versus observation in patients with locally advanced nonsmall cell lung cancer: primary analysis of radiation therapy oncology group study RTOG 0214. J Clin Oncol. $2011 ; 29: 272-8$.

11. Sun A, Bae K, Gore EM et al. Phase III trials of prophylactic cranial irradiation compared with observation in patients with locally advanced non-small cell lung cancer: neuro-cognitive and quality-of-life analysis. J Clin Oncol. 2011; 29: 279-86.

12. Viney R, Lancsar E et al. Discrete choice experiments to measure consumer preferences for health and healthcare. Expert Rev Pharmaco Outcomes Res. 2002; 2: 319-326.

13. Viney R, Savage E, Louviere J. Empirical investigation of experimental design properties of discrete choice experiments in health care. Health Economics. 2005; 14: 349-362.

14. Johnson FR, Lancsar E, Marshall D, et al. Constructing experimental designs for discrete-choice experiments: Report of the ISPOR Conjoint Analysis Experimental Design Good Research Practices Task Force. Value Health 2013; 16:3-13.

15. www.euroqol.org/eq-rd-products/eq-rd-rl.htm) 
McFadden D and Train K. Mixed MNL models for discrete response. Journal of Applied Econometrics. 2000; 15: 447-470.

17. Hensher DA, Rose JM, Greene WH. Applied Choice Analysis: A Primer, New York: Cambridge University Press, 2005

18. Mehta MP, Rodrigus $\mathrm{P}$, Terhaard $\mathrm{CH}$ et al. Survival and neurologic outcomes in a randomised trial of motexafin gadolinium and whole brain radiation therapy in brain metastases. J Clin Onc. 2003. 21: 2529-2536

19. Cox JD, Stanley K, Petrovich Z et al. Cranial irradiation in cancer of the lung of all cell types. JAMA. 1981; 245: 469-472

20. Unasawadi T, Valdivieso M, Chen T et al. Role of elective brain irradiation during combined chemoradiotherapy for limited disease non-small cell lung cancer. J Neurooncol. 1984; 2: 253-259.

21. Russell AH, Pajak TE, Selim HM et al. Prophylactic cranial irradiation for lung cancer patients at high risk for development of cerebral metastasis: results of a prospective randomised trial conducted by the Radiation Therapy Oncology Group. Int J Radiat Oncol Biol Phys. $1991 ; 21: 637-643$.

22. Pöttgen C, Eberhardt W, Grannass A et al. Prophylactic cranial irradiation in operable stage IIIA non-small cell lung cancer treated with neoadjuvant chemoradiotherapy: results from a German multicentre randomised trial. J Clin Oncol. 2007; 25: 4987-92.

23. Miller TP, Crowely JJ, Mira J et al. A randomised trial of chemotherapy and radiotherapy for stage III non-small cell lung cancer. Cancer Therapeutics. 1998; 4:229-236.

24. McQuellon RP, Muss HB, Hoffman SL, Russell G, Craven B, Yellen SB. Patient preferences for treatment of metastatic breast cancer: a study of women with early-stage breast cancer. J Clin Oncol. 1995; 13: 858-68.

25. Duric V, Stockler M. 2001 Patients 'preferences for adjuvant chemotherapy in early breast cancer: a review of what makes it worthwhile? Lancet Oncol. 2001 2: 691-7.

26. Gondi V, Tome WA, Mehta M. Why avoid the hippocampus? A comprehensive review. Radiotherapy and Oncology. $2010 ; 97: 370-376$.

27. Gondi V, Pugh SL, Tome WA et al. Preservation of memory with conformal avoidance of the hippocampal neural stem-cell compartment during whole-brain radiotherapy for brain metastases (RTOG 0933): A phase II multi-institutional trial. J Clin Oncol. 2014 ; 32 : 3810 -3816.

28. Tsai P, Yang C, Chuan C et al. Hippocampal dosimetry correlates with the change in neurocognitive function after hippocampal sparing during whole brain radiotherapy: a prospective study. Radiation Oncology 2015; 10: 253-268

29. Paumier A, Cuenca X, Le Péchoux C. Prophylactic cranial irradiation in lung cancer. Cancer Treatment Reviews. 2011; 37: 261-265.

30. Gondi V, Paulus R, Bruner D et al. Decline in Tested and Self-reported cognitive functioning after prophylactic cranial irradiation for lung Cancer: Pooled secondary analysis of Radiation therapy Oncology Group Randomised Trial 0212 and 0214. International Journal of Radiation Oncology Biology and Physics. 2013; 86: 656-664.

31. Whole-Brain Radiation Therapy with or without Hippocampal Avoidance in Treating Patients with Limited Stage or Extensive stage Small Cell Lung Cancer. NRG-CC 003. ClinicalTrials.gov Idenitifier: NCT02635009. Available at https://clinicaltrials.gov. 
32. Memantine Hydrochloride and Whole-Brain Radiotherapy with or without hippocampal avoidance in reducing neurocognitive decline in patients with brain metastases. NRG-CC 001. ClinicalTrials.gov Identifier: NCT02360215. Available at https://clinicaltrials.gov.

33. Gondi V, Tome W, Marsh J et al. Estimated risk of perihippocampal avoidance during whole brain radiation therapy: safety profile for RTOG 0933 . Radiother Oncol 2010; 95: 327-31

34. Marsh J, Herskovic A, Gielda B et al. Intracranial metastatic disease spares the limbic circuit: a review of 697 metastatic lesions in 107 patients. Int J Radiat Oncol Biol Phys 2010; 76: 504-12.

35. Harth s, Abo-Madyan Y, Zhen L et al. Estimation of intracranial failure risk following hippocampal-sparing whole brain radiotherapy. Radiother Oncol 2013; 109: 152-158.

36. Kimman M, Dellaiert B, Boersma L et al. Follow-up after treatment for breast cancer; one strategy fits all? An investigation of patient preferences using a discrete choice experiment. Act Oncol 2010; 49; 328-337

37. Benning $T$, Kimman M, Dirksen $C$ et al. Combining individual-level discret choice experiemtn estimates and costs to inform health care management decisions about customised care: the case of follow-up strategies after breast cancer treatment. Value in Health 2012; 15: 680-689. 
Figure 1: Example of a choice situation from the DCE (Pre-treatment Survey, Choice Situation 1) (Supplementary)

Figure 2: Implied importance weights for improvement in PCl treatment attributes

Table 1: Attributes and Levels for the DCE choice sets

Table 2: Sample characteristics of participants, $n=54$

Table 3: MXL Model Results, Pre and Post Treatment (Supplementary)

Table 4: Implied importance weights for improvement in PCl treatment attributes (Supplementary)

Table 5: Estimate for probability of $\mathrm{PCl}$ uptake in selected hypothetical scenarios, pre and post treatment

Table 6: Estimate for probability of PCI uptake in selected hypothetical scenarios, pre and post treatment (aligned with clinical evidence from RCT) 